\title{
Atrial septal aneurysm is not always linked to right-left shunt and cardio embolic risk
}

\author{
Sarah Mangiafico ${ }^{1}$, Ines Paola Monte ${ }^{1}$, Vincenzo Lavanco ${ }^{1}$, Lucio Raffaello Tropea ${ }^{1}$, \\ Antonio Andrea Arcidiacono ${ }^{1}$, Maria Concetta di Pasqua ${ }^{1}$, Letizia Santonoceto ${ }^{1}$, \\ Giovanni Millan ${ }^{1}$, Corrado Tamburino ${ }^{1,2}$
}

${ }^{1}$ Dept, Cardio-Thoraco-Vascular, AOU Policlinic University of Catania, Catania, Italy

${ }^{2}$ Excellence through Newest Advances (ETNA) Foundation, Catania, Italy

Email address:

inemonte@unict.it(I. P. Monte), sarah.mangiafico4@gmail.com(S. Mangiafico)

\section{To cite this article:}

Sarah Mangiafico, Ines Paola Monte, Vincenzo Lavanco, Lucio Raffaello Tropea, Antonio Andrea Arcidiacono, Maria Concetta di Pasqua, Letizia Santonoceto, Giovanni Millan, Corrado Tamburino. Atrial Septal Aneurysm Is Always Linked To Right-Left Shunt and Cardio embolic Risk. Clinical Medical Research. Vol. 2, No. 4, 2013, pp. 48-52. doi: 10.11648/j.cmr.20130204.12

\begin{abstract}
Atrial septal aneurysm when associated to other cardiac abnormalities, such as patent foramen ovale, Chiari network or Eustachian Membrane, is a well-known recognised risk factor for cerebral embolism. Aim of study is to assess if isolated atrial septal aneurysm could be considered statistically related to cryptogenic stroke, considering the strong clinical impact that this association could have on these patients outcome. 579 patients were investigated for a suspected patent foramen ovale using transthoracic echocardiography, in order to evaluate the presence of atrial septal aneurysm and to identify other potential cardiac embolic sources. The patients with atrial septal aneurysm underwent transcranic echodoppler and transoesophageal echocardiography examination with contrast solution during Valsalva manoeuvre.209/579 (36\%) patients where we made diagnosis of atrial septal aneurysm. In this cohort of patients transcranic echodoppler showed a patent foramen ovale in 114/209 (54\%), whereas transoesophageal echocardiography in 111/209 (53\%); 6/114 (5\%) patients with a positive transcranic echodoppler had a normal transoesophageal echocardiography examination and 3/95 (3\%) with normal transcranic echodoppler had a positive transoesophageal echocardiography. Transcranic echodoppler vs. transoesophageal echocardiography sensibility was $97 \%$ and specificity $94 \%$. Only $13 / 98$ (13\%) of patients with isolated atrial septal aneurysm and 23/111 (26\%) with patent foramen ovale had history of cryptogenic stroke. According to our data, isolated atrial septal aneurysm could not be considered an independent risk factor for cryptogenic stroke.
\end{abstract}

Keywords: Atrial Septum Aneurysm, Stroke, Echocardiography

\section{Introduction}

Atrial septal aneurysm (ASA) is a rare deformity of the atrial septum that bulges into the right or the left atrium with uncertain clinical significance. It has been reported in $1 \%$ of consecutive autopsies and in about $4.9 \%$ of patients undergoing Transoesophageal Echocardiography (TEE) performed for any clinical condition suggestive of the presence of emboli [1-4]. With the use of Transthoracic echocardiography (TTE) and TEE, ASA can also be defined when the basal width of the aneurysm is more than $15 \mathrm{~mm}$ and the excursion of the dilated septum beyond the plane of the residual atrial septum is at least $10 \mathrm{~mm}$ [5].

ASA can be associated with right-left shunt (R-Ls) and patent foramen ovale (PFO) and it is unclear if when isolated can be a cause of embolism, on the contrary the asso- ciation of ASA with other cardiac abnormalities such as Chiari network $(\mathrm{CN})$ or Eustachian Membrane (EM) is a well-known recognised risk factor for embolic events, such as cryptogenic stroke and cryptogenic stroke recurrence [5, 6]. Furthermore, in young patients with embolic events the correlation of ASA and R-Ls has been reported in up to $97 \%$ of cases [7]. Some authors have associated even an isolated ASA with ischemic stroke in view of its potential embolic role [3, 8, 9]. It has also been demonstrated a higher percentage of ASA in patients with cerebral embolism of unknown origin $(7.9 \%)$ compared to a large matched population-based (thus not pre-selected) control group $(2.2 \%)$ [10].

The aim of our study is to assess if isolated ASA could be considered statistically related to cryptogenic stroke, considering the strong clinical impact that this association 
could have on these patients outcome.

\section{Methods}

Our prospective study included 579 patients (mean age 46 years, range $12-70$ ) who underwent investigations for a suspected PFO from October 2009 to October 2011, in the Cardiology Unit of the Cardio-Thoraco-Vascular Department, AOU Policlinic University of Catania. All the patients underwent: clinical evaluation, measurement of blood pressure and a standard 12-lead ECG, in order to exclude arrhythmic clinical conditions such as atrial fibrillation (AF) and supraventricular tachyarrhythmia, which on their own can cause embolism. All patients with history of cryptogenic stroke, an echographic evaluation of carotid arteries were performed with the aim to assess potential causes of the stroke.

In accordance with EAE recommendations, [11] TTE was the first line investigation used in order to evaluate the presence of ASA and to identify other potential cardiac embolic sources. Only patients with ASA underwent transcranic echodoppler (TCD) and TEE examination with contrast solution (cTCD and cTEE).
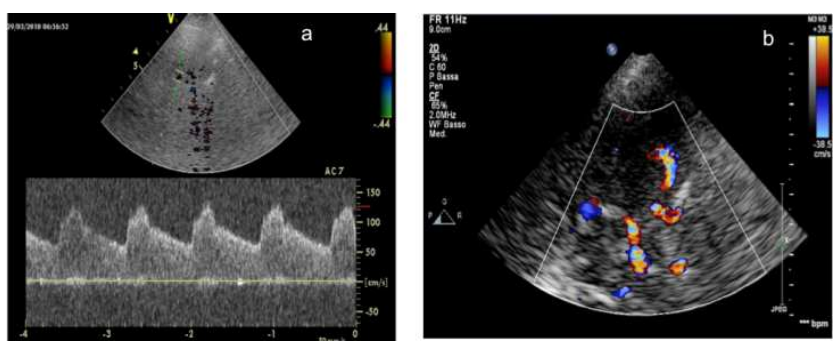

Figure 1. Transcranic echodoppler with flow pattern in the cerebral artery (a) and colordoppler visualization (b) of middle, posterior and anterior cerebral arteries

cTCD (Fig.1) was performed with an Acuson Sequoia (3V2C probe) and all the examinations were stored in Digital imaging. We performed Doppler studies of the middle, anterior and posterior (segments P1-P2) cerebral artery through left and right temporal projection and when these imagines were considered unsatisfactory an occipital projection was used to clarify. The exam included an intravenous administration of three to six samples of contrast medium in a brachial vein through a 20 -gauge cannula-needle. The contrast medium was obtained by forcefully mixing a solution between two $10 \mathrm{ml}$ syringes, one of which contained $9 \mathrm{ml}$ of saline solution and the other $1 \mathrm{ml}$ of air. Patient was requested to start Valsalva manoeuvre (VM) for 5 seconds at the same moment of the infusion of the contrast medium. The VM was considered effective if it was able to reduce the flow in the cerebral artery. The passage of microbubbles (PM) was counted for 25 seconds after the end of VM [12].

cTEE examination (Fig. 2) was performed with a $5 \mathrm{MHZ}$ multiplane probe or with a $3.5-5 \mathrm{MHz}$ multiplane, fasting for $3 \mathrm{hrs}$ and with the use of topical oral and pharyngeal anesthetizing agents (lidocaine spray 10\%). Color Doppler was used to help the presence of shunts detection. The interatrial septum was visualized in the transverse midesophageal four-chamber view and in the longitudinal biatrial-bicaval view. The diagnosis of R-Ls was done when at least three microbubbles passed in the left atrium during the subsequent cardiac cycles. The suspected size of PFO was determined by the number of microbubbles that passed from right atrium to left atrium: a small PFO was defined with the passage of 3 to 9 bubbles through the foramen; medium with 10 to 30 bubbles, and large with more than 30 bubbles [13-15].

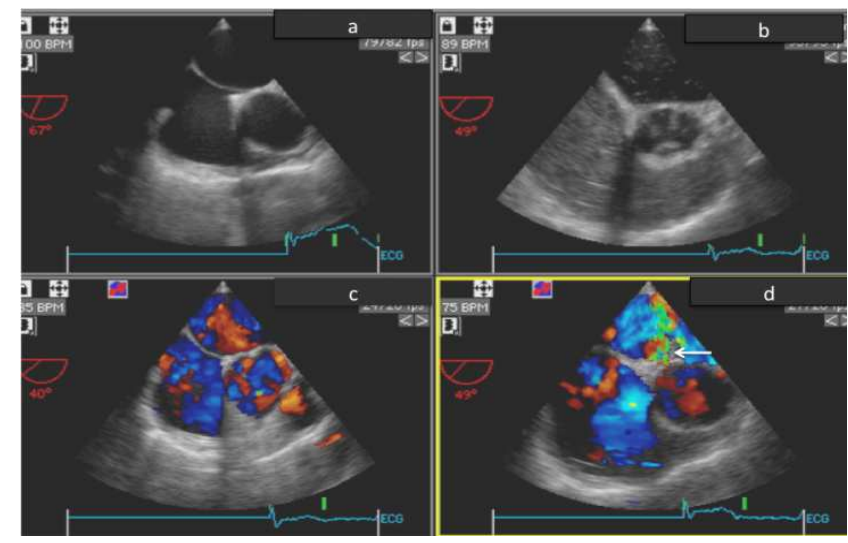

Figure 2. Transesophageal Ecocardiography using to evaluate the rightleft shunts through atrial septum during Valsalva manoevre with microbubbles in left atrium (b) and color aliasing across septum (d, arrow)

During the examination VM was requested at the time of injection of forceful mixed solution with the same modalities previously described for cTCD. In this case, VM was considered positive in the presence of a reduction of the diameter of the right atrium and ventricle cavity and when a major extension of the atrial septum was visible through the left atrium.

This study complied with the Declaration of Helsinki. It had ethical committee approval and all the patients gave consent to use their data.

\subsection{Statistical Analysis}

The results were subjected to statistical analysis using tools for the assessment of risk factors such as calculation of Odds, Absolute Risk (AR), and Relative Risk (RR), the ODDS Ratio (OR) and Yates's chi squared test was used.

\section{Results}

In our series we have studied 579 patients (age 39+13 years): all clinical presentation and cardiovascular risk factors of these patients are summarized in Table 1:

We had 418/579 (72\%) female patients and 161/579 (28\%) male patients. $306 / 579(53 \%)$ of the patients reported history of cardiovascular disease. Smoking, diabetes and dyslipidemia were a risk factor in 208/579 (36\%), $133 / 579$ 
Table 1. Clinical characteristics of patients

\begin{tabular}{lcc}
\hline & $\begin{array}{c}\text { Patients number (total } \\
\text { 579) }\end{array}$ & \% \\
\hline Male/Female & $161 / 418$ & $28 / 72$ \\
Migraine with Aura & 222 & 38 \\
Cryptogenic Stroke & 144 & 25 \\
Vertigo & 82 & 14 \\
Prior diagnosis of ASA & 131 & 23 \\
History of CVD in I degree relatives & 306 & 53 \\
Smoke & 208 & 36 \\
Diabetes & 133 & 23 \\
Dyslipidemia & 197 & 34 \\
Trombophilia & 41 & 7 \\
\hline
\end{tabular}

$(23 \%)$ and $197 / 579(34 \%)$ respectively. Positive trombophilia screen (which included antiphospolipids antibodies, protein $\mathrm{C}$ and $\mathrm{S}$ deficit and Factor $\mathrm{V}$ deficit) was reported positive in $41 / 579(7 \%)$ patients. $222 / 579(38 \%)$ patients complained of migraine with aura, $82 / 579$ (14\%) of vertigo and $131 / 579(23 \%)$ patients were with no symptom, but with a previous detection of ASA. 144/579 (25\%) had in their past medical history at least one episode of cryptogenetic stroke.

$209 / 579$ (36\%) patients where we made diagnosis of ASA using ETT. Afterwards, all patients with ASA underwent cTCD and cTEE (Fig. 3).

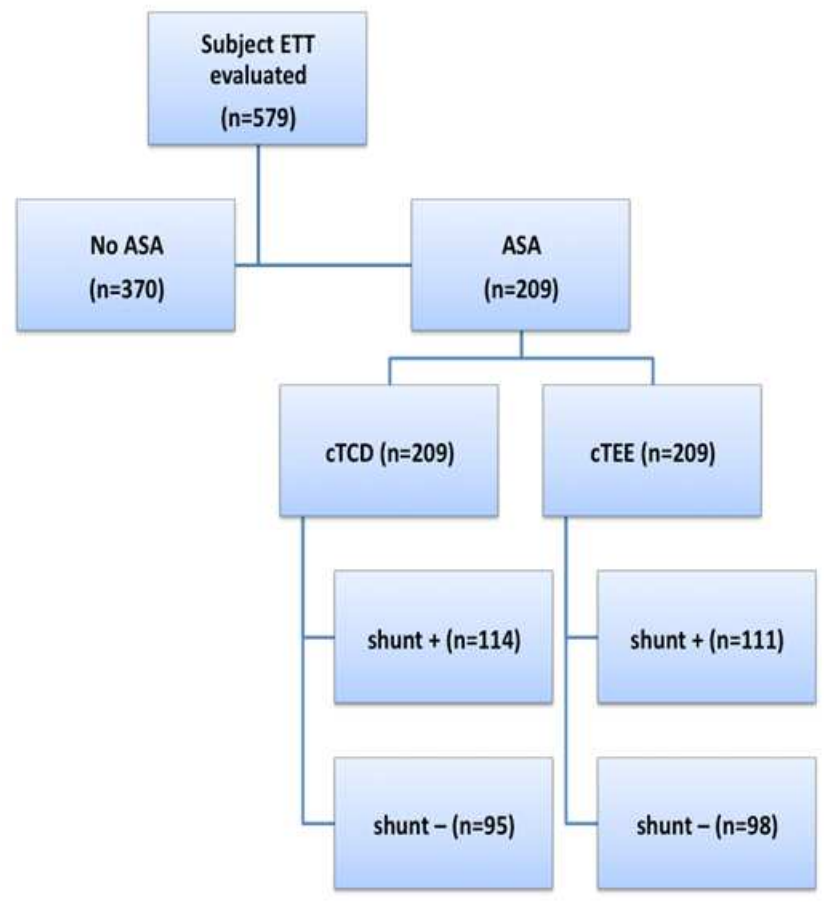

Figure 3. Evidence of right-left atrial shunt in patients with atrial septal aneurysm (ASA) at Transthoracic Ecocardiography (TTE), evaluated by Contrast Transcranic echodoppler (cTCD) or Contrast Transesophageal Ecocardiography (cTEE)
Table 2. Accuracy of cTCD vs. cTEE in detecting patent foramen ovale

\begin{tabular}{|c|c|c|c|c|c|c|c|}
\hline & $\begin{array}{l}\text { c-TEE: } \\
\text { PFO + }\end{array}$ & $\begin{array}{l}\text { C-TEE: } \\
\text { PFO - }\end{array}$ & $\begin{array}{l}\text { Sensitivi- } \\
\text { ty }(\%)\end{array}$ & $\begin{array}{c}\text { Specificity } \\
(\%)\end{array}$ & $\begin{array}{l}\text { PPV } \\
(\%)\end{array}$ & $\begin{array}{l}\text { PNV } \\
(\%)\end{array}$ & $\begin{array}{c}\text { Accura- } \\
\text { cy (\%) }\end{array}$ \\
\hline $\begin{array}{l}\text { cTCD: } \\
\text { PFO + } \\
\text { cTCD: } \\
\text { PFO - }\end{array}$ & 108 & $\begin{array}{c}6 \\
92\end{array}$ & 97 & 94 & 95 & 97 & 96 \\
\hline
\end{tabular}

cTCD: contrast transcranic echodoppler; cTEE: two-dimensional contrast transesophageal echocardiography; PFO: patent foramen ovale; PPV: predictive positive value; PNV: predictive negative value

cTCD examination in this cohort of patients showed a PFO in 114/209 (54\%) and among these 18/114 (16\%) was a category 1 shunt (PFO small), 12/114 (10\%) was a category 2 shunt (PFO mild) and 84/114 (74\%) was a category 3 shunt (PFO large). 95/209 (46\%) had normal c-TCD exam. cTEE examination showed: 6/114 (5\%) patients with a positive cTCD had a normal cTEE examination. On the contrary, 3/95 (3\%) patients with normal cTCD examination had a positive cTEE. In summary, when cTEE examination was used we had 111/209 (53\%) with PFO; of these patients $12 / 111(11 \%)$ had a small shunt, $9 / 111(8 \%)$ had an mild shunt and 90/111 (81\%) had a large shunt. $98 / 209(46,8 \%)$ patients had an isolated ASA. cTCD vs cTEE sensibility was $97 \%$, specificity $94 \%$ and accuracy 96\% (Table 2).

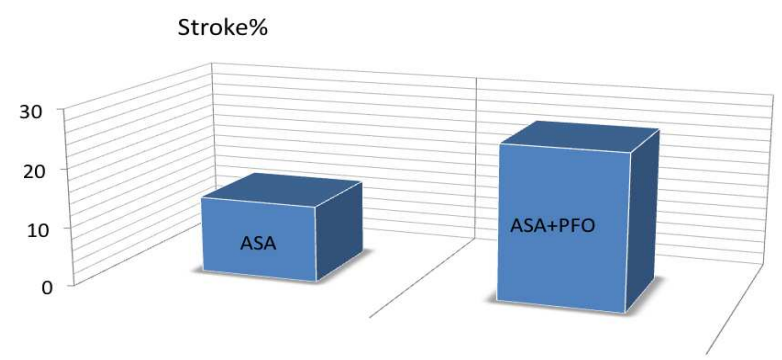

Figure 4. Percentage of patients with history of cryptogenic stroke in group with atrial septal aneurysm (ASA) and in group with ASA and $A S A+P F O$

Of the 98/209 patients with isolated ASA only $13(13 \%)$ patients had history of cryptogenic stroke with an OR 0.28 (CI: $0.14-0.57)(\mathrm{p}<0.001)$, whereas $23 / 111(26 \%)$ with patent foramen ovale patients (Fig. 4). Patients with isolated ASA and stroke there was a strong male vs female prevalence ( 9 male/ 4 female).

\section{Discussion}

It may be very challenging to study the real impact of isolated ASA in cryptogenic stroke, because it is often associated with PFO presence and it is not very common in general population. Moreover, there are controversial literature data on its emboligenic potential when isolated, both in promoting the income of a "brand new" cryptogenic stroke, stroke recurrence or to worsen the level and gravity of white matter lesions in stroke patients; while it is quite 
clear its role as an adjunctive risk factor in presence of R-L shunt $[3,8-11,16]$.

In agreement with literature, in our series we did not find significant differences in the prevalence of PFO in relation to gender [17] while we found high male/female prevalence (70\% vs 30\%) in patients with isolated ASA and stroke.

In our series we have found a quite higher presence of ASA (36\% of our patients) in comparison with general population presence $(2,2 \%)$ [5]. This could be easily explained by considering that many of our patients were sent to our observation to assess the presence of a R-L shunt after a TTE performed for other reasons which then revealed the presence of ASA.

There is a direct relationship between the size of a shunt through a PFO and the risk of illness PFO related. Moreover, the risk of stroke appears to be increased in the presence of defects that direct flow towards a PFO, for example in patients with a prominent EM or hemodynamic changes that increase right-sided pressure (such as a large pulmonary embolism) which can promote a $\mathrm{R}-\mathrm{L}$ shunt.

If there is a R-L shunts suspicion, TTE should be considered the first line examination, which could at the same time help to diagnose the presence of ASA or atrial potential cardiogenic embolic factors.

Currently, TEE associated with intravenous injection of agitated saline contrast represents the reference imaging technique for anatomical and functional definition of the interatrial septum and measurement of PFO size [12]. However, TEE is a semi-invasive procedure burdened with patient discomfort, procedural risk, and significant time consumption.

In previous study, Monte and coll. [18] showed a relationship between the size of the PFO and accuracy of 3D transthoracic Echocardiography, with false-negative cases limited to patients with small size of PFO $(<2 \mathrm{~mm})$ and poor acoustic windows.

Several studies have shown that the CTCD is a safe technique, with high sensitivity and specificity and a good alternative to the cTEE for R-L shunt diagnosis (sensitivity $92 \%$, specificity $97 \%$ for cTCD versus $100 \%$ for cTEE if the latter is accepted as the gold standard) $[4,12-13,19]$. Even though cTCD could be considered a good alternative to c-TEE, in patients with ASA we still believe that a cTEE should also performed, because it allows visualizing directly the septal anatomy and confirming the ASA presence and it can quite easily distinguish between the various causes of interatrial shunt (PFO vs. DIA) with colour flow mode.

In our series we did not found a statistical correlation between isolated ASA and cryotogenic stroke; this is a quite interesting result because it is demonstrated that, even though ASA could be considered as an emboligenic condition by itself, it is not statistically related to stroke income when there is no a demonstrated R-L shunt. Moreover in our series only $13 / 579(2 \%)$ had a neurological ischemic event with an ASA but with no R-L shunting.

According to our results, we can state that all patients with ASA, particularly patients with history of cryptogenic stroke, should undergo a cTEE to detect PFO presence and a carefully examination of the septal anatomy to detect the shunt direction should be performed.

If PFO is present in absence of other potential stroke causes we usually counselling the patients in the treatment of the defect. In addition, if there is no a R-L shunt the patient should be resent to a specialist neurologic centre to have a diagnostic work-up to look for other causes of cryptogenetic stroke.

\section{Conclusions}

According to our data, we can conclude that isolated ASA could not be considered an independent risk factor for cryptogenic stroke; all patients with stroke and ASA should undergo a full diagnostic workup to assess the presence of R-L shunting with a mandatory use of cTEE. If R-L shunt presence is not confirmed by cTEE, these patients should be referred for a neurologist opinion and a diagnostic stroke workup in order to look for other cryptogenetic stroke causes is warrant.

\section{References}

[1] Silver MD, Dorsey JS. Aneurysms of the septum primum in adults. Arch Pathol Lab Med 1978;102:62-5.

[2] Gondi B, Nanda NC.Two dimensional echocardiographic features of atrial septal aneurysms. Circulation 1981; 63: $452-457$

[3] Belkin RN, Kisslo J. Atrial septal aneurysm: recognition and clinical relevance. Am Heart J 1990; 120: 948-957

[4] Pearson AC, Labovitz AJ, Tatineni S, Gomez CR. Superiority of transesophageal echocardiography in detecting cardiac source of embolism in patients with cerebral ischemia of uncertain etiology. J Am Coll Cardiol 1991; 17: 66-72

[5] Ghosh S, Ghosh AK, Ghosh SK. Patent foramen ovale and atrial septal aneurysm in cryptogenic stroke. Postgrad Med J 2007; 83: 173-177

[6] Davison P, Clift PF, Steeds RP. The role of echocardiography in diagnosis, monitoring closure and post-procedural assessment of patent foramen ovale. Eur J Echocardiogr 2010; 11 (10): i27-34

[7] Mattioli AV, Aquilina M, Oldani A, Longhini C, Mattioli G. Atrial septal aneurysm as a cardioembolic source in adult patients with stroke and normal carotid artery. A multicentre study. Eur Heart J 2001; 22: 261-268

[8] Gallet B, Malergue MC, Adams C, Saudemont JP, Collot AM, Druon MC, Hiltgen M. Atrial septal aneurysm a potential cause of systemic embolism. Br Heart J 1985; 53: 292 297

[9] Razaq M, Pariar RK, Saini G. Atrial septal aneurysm and stroke Ann Pediatr Cardiol 2012; 5 (1): 98-99

[10] Force M, Massabuau P, Larrue PV. Prevalence of atrial septal abnormalities in older patients with cryptogenic 
ischemic stroke or transient ischemic attack. Clin Neurol Neurosurg 2008; 110 (8): 779-783

[11] Pepi M, Evangelista A, Nihoyannopoulos P, Flachskampf FA, Athanassopoulos G, Colonna P, Habib G, Ringelstein E, Sicari R, Zamorano JL. Recommendations for echocardiography use in the diagnosis and management of cardiac sources of embolism. Eur J Echocard 2010; 11, 461476

[12] Blersch WK, Draganski BM, Holmer SR, Koch HJ, Schlachetzki F, Bogdahn U, Hölscher T . Transcranial Duplex Sonography in the Detection of patent foramen ovale. Radiology 2002; 225: 693-699

[13] Mas JL, Arquizan C, Lamy C, Zuber M, Cabanes L, Derumeaux G, Coste J. Recurrent cerebrovascular events associated with patent foramen ovale, atrial septal aneurysm, or both. N Engl J Med 2001; 345: 1740-1746

[14] Schuchlenz HW, Weihs W, Beitzke A, Stein JI . Transesophageal Echocardiography for Quantifying Size of Patent Foramen. Stroke 2002; 33:293-296

[15] Mangiafico S, Scandura S, Ussia GP, Privitera A, Capodanno D, Petralia A, Tamburino C. Transesophageal echocardi- ography and transcranial color-doppler: indipendent or complementary diagnostic tests for cardiologists in the detection of patent foramen ovale? J Cardiovasc Med 2009; 10:143-148.

[16] Agmon Y, Khandheria BK, Meissner I, Gentile F, Whisnant JP, Sicks JD, O'Fallon M, Covalt J, Wiebers D, Seward JB . Frequency of atrial septal aneurysms in patients with cerebral ischemic events. Circulation 1999; 99: 1942-1944

[17] Ueno Y, Shimada Y, Tanaka R, Miyamoto N, Tanaka Y, Hattori N, Urabe T. Patent Foramen Ovale with Atrial Septal Aneurysm May Contribute to White Matter Lesions in Stroke Patients. Cerebrovasc Dis 2010; 30: 15-22

[18] Monte I, Grasso S, Licciardi S, Badano LP. Head-to-head comparison of real-time three-dimensional transthoracic echocardiography with transthoracic and transesophageal two-dimensional contrast echocardiography for the detection of patent foramen ovale. Eur J Echocardiogr 2010; 11: $245-249$

[19] Cruz-Gonzales I, Solis J, Inglessis-Azuaije I, Palacios IF. Patent Foramen Ovale: current state of art. Rev Esp. Cardiol 2008; 61 (7): 738-751. 